\title{
A Novel Homozygous Nonsense Variant in BICD2 Underlies Hereditary Spastic Paraplegia Complex Type
}

\author{
Sajida Rasool ${ }^{1}$, Saba Irshad ${ }^{1 *}$, Neelam Saba ${ }^{1}$, Mehak Fiaz ${ }^{1}$, \\ Muhammad Sajid Hussain ${ }^{2}$, Muhammad Wajid Hussain ${ }^{3}$ and Peter Nürnberg ${ }^{2}$ \\ ${ }^{1}$ Institute of Biochemistry and Biotechnology, University of the Punjab, \\ Quaid-I-Azam Campus, Lahore, 54590 \\ ${ }^{2}$ Cologne Center for Genomics, University of Cologne, D-50931 Cologne, Germany \\ ${ }^{3}$ Department of Zoology, Okara University, Okara, Pakistan
}

\begin{tabular}{l} 
Article Information \\
\hline Received 22 January 2019 \\
Revised 22 May 2019 \\
Accepted 18 June 2019 \\
Available online 28 January 2020 \\
Authors' Contribution \\
SI, PN and MSH conceived and \\
designed the study. SR, NS and MSH \\
acquired and analyzed the data. SR \\
contributed to drafting the text and \\
preparing the figures. WH and NS \\
helped in recruiting families. \\
Key words \\
Hereditary Spastic Paraplegia (HSP), \\
BICD2, Nonsense mutation, Spasticity
\end{tabular}

\begin{abstract}
A B S T R A C T
Hereditary spastic paraplegia (HSP) are a group of genetically and clinically diverse neurodegenerative ailments with distinguishing feature of lower limbs spasticity and upper motor neuron degeneration. BICD2 is an adaptor protein which regulates the cellular trafficking of cargo molecules crucial for motor neuron growth and maturation. In present study, a Pakistani family of HSP penetrating in autosomal recessive pattern was ascertained. Patients presented spasticity and stiffness of upper and lower limbs, severe microcephaly, dysphagia, no speech, hearing loss and seizures. Genome wide linkage analysis and whole exome sequencing revealed a novel homozygous nonsense mutation (c.204T $>\mathrm{G}$ ) in BICD2 gene which was predicted to yield a truncated protein product ( $\mathrm{p}$. Glu68*). This is first nonsense mutation being reported causing HSP with complex clinical features and early onset. Further, functional exploration will be required for genotype phenotype correlation.
\end{abstract}
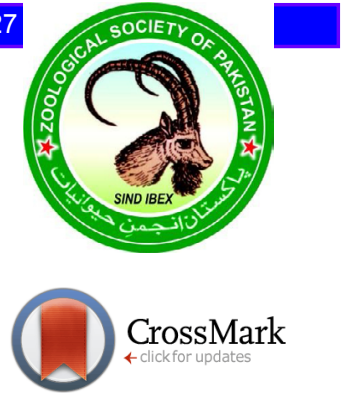

\section{INTRODUCTION}

$\mathrm{H}^{-1}$ ereditary spastic paraplegia (HSP) are a group of neurodegenerative or neurodevelopmental disorders of clinically and genetically heterogeneous nature. The most established pathomechanism of neurodegeneration involves the progressive distal axonopathy of the longest motor fibers that encompasses the corticospinal tract and posterior column (Klebe et al., 2015). The distinguishing neurological and clinical feature is the spasticity of the lower limbs, weakness and hyperreflexia. However, HSP patients present a variety of neurological and nonneurological symptoms (Blackstone et al., 2011; Parodi et al., 2017). This disorder is classified into pure and complex forms, depending on the clinical features they present. Pure HSP form includes lower limb spasticity, which is sometimes accompanied with bladder spasticity and hyperreflexia, while complex HSP exhibits other associated neurological and non-neurological features such as seizures, amyotrophy, ataxia, cognitive impairment, deafness, ophthalmic dysfunction, facial deformities, orthopedic abnormalities, and digestive reflux.

\footnotetext{
Corresponding author: saba.ibb@pu.edu.pk 0030-9923/2020/0002-0727 \$ 9.00/0

Copyright 2020 Zoological Society of Pakistan
}

A number of molecular mechanisms such as abnormal intracellular trafficking, disturbed organelle morphogenesis, axonal transport dysfunction, lipid metabolism disturbance, mitochondrial dysfunction and dysregulated pigmentation are proposed to be implicated in pathogenicity of the disease (Salinas et al., 2008; Nagle et al., 1996). To date, 59 causative genes (https:// www.ncbi.nlm.nih.gov/books/NBK1509/) have been identified involving all mode of inheritance. These include autosomal dominant, autosomal recessive, X-linked and maternal mitochondrial transmission (Giudice et al., 2014; Finsterer et al., 2012).

Bicaudal D, Drosophila homologue 2 (BICD2) has been identified as causative gene of autosomal dominant spinal muscular atrophy with lower extremity predominance 2 (SMALED2) and hereditary spastic paraplegia (HSP) (Synofzik et al., 2014; Neveling et al., 2013; Oates et al., 2013; Peeters et al., 2013; Rossor et al., 2015). BICD2 regulates the trafficking of key cellular cargoes like mRNA, Golgi, and secretary vesicles by interacting with dynein-dynactin complex and small GTPase RAB6. A flawless retrograde and anterograde transport along microtubules is very crucial for motor neuron development and functional integrity (Matanis et al., 2002; Hoongenraad et al., 2001). 
In this study, we report a family of congenital onset, complex hereditary spastic paraplegia with autosomal recessive mode of inheritance. We identified a protein truncation mutation in BICD2 after whole exome sequencing (WES) and Sanger sequencing techniques.

\section{MATERIALS AND METHODS}

\section{Study material}

This study was commenced with the approval from bioethics committee of University of the Punjab. Lahore. Informed consent was obtained from the patient's parents. A family with two affected individuals (V-1 and V-2) presenting hereditary spastic paraplegia of complex type and severe microcephaly was ascertained from region of north-eastern Pakistan to explore the cause of the disorder. Blood samples from two affected and six unaffected individuals were obtained in ethylenediaminetetra acetic acid (EDTA) containing BD vacutainers ${ }^{\circledR}$ (BD-Plymouth, UK). Genomic DNA was extracted using salting out method (Helms, 1990).

\section{Linkage analysis}

Genomic DNA from four individuals (two affected and the parents) were submitted for genome-wide linkage analysis using the Axiom Precision Medicine Research Array from Affymetrix (Santa Clara, CA). LOD score was calculated with the reduced marker panels of $25 \mathrm{~K}$ and $100 \mathrm{~K}$ using programme ALLEGRO (Gudbjartsson et al., 2000). Linkage analysis was performed assuming autosomal recessive mode of inheritance, full penetrance, consanguinity and allele frequency of 0.0001 . Mendelian errors were detected using PedCheck (O'Connell and Weeks, 1998).

\section{Whole exome sequencing}

Whole exome sequencing of one affected individual (V-1) was performed using the Agilent (Santa Clara, CA) version 6 enrichment kit and the Illumina HiSeq 4000 sequencing system (paired-end reads, $2 \times 75 b p$ ). The exome data was filtered and analysed using Cologne Center for Genomics in house database VARBANK (http:// varbank.ccg.uni-koeln.de) as described (Szczepanski et al., 2016). Exome data was also explored for variants in causative genes of other clinical symptoms exhibited by patients like microcephaly, hearing loss, intellectual disability and speech impairment. Variant prioritization was done with relation to phenotype using wANNOVAR (http://wannovar.wglab.org) and Phenolyzer (http:// phenolyzer.wglab.org). The prioritized variants were analysed using bioinformatic tools for pathogenicity prediction.

\section{Co-seggregation analysis}

A variant identified in $B I C D 2$ as possible cause of disease phenotype was validated for co-segregation using Sanger sequencing. Sequencing primers (Forward primer: 5'-TCGAGGAGAAGCACCAGCTCAAG-3'; Reverse primer: 5'-CTGCATTAGCGGCGTCTGCAAC-3') were designed using Primer 3 software (https://bioinfo. ut.ee/primer3-0.4.0/primer3/). BigDye ${ }^{\circledR}$ Terminator v1.1 Cycle sequencing kit (Applied Biosystem) was used for sequencing of amplified PCR product on ABI 3730 genetic analyzer (Applied Biosystem). Sequencing data was analysed on DNA Star (Lasergene).

\section{RESULTS}

\section{Patients}

Two affected individuals were born to the normal parents who were second cousins. Analysis of pedigree (Fig. 1A) confirmed an autosomal recessive mode of inheritance. Both patients were reported to be affected by birth. Patient V-1 was a 3 years old female at the time of examination and her brother ( $\mathrm{V}-2)$ was 1.5 years old (Fig. 1B). Birth of both affected individuals was after full term pregnancy and without complication. Both patients exhibited clinical features of hereditary spastic paraplegia with spasticity and stiffness of lower as well as upper limbs and severe microcephaly with reduced head circumference of -10 and -11 SD. Both patients presented hearing impairment, no speech, dystonia, dysphagia and seizures. They often had screaming bouts. They could not sit or stand. Individual V-1 has normal height of $91.4 \mathrm{~cm}$ while the boy $\mathrm{V}-2$ had slightly short stature of $73.6 \mathrm{~cm}$ $(-2.5 \mathrm{SD})$.

\section{Mutation identification}

Genome wide linkage analysis using $25 \mathrm{~K}$ panel resulted in two peak areas reaching the theoretical maximum LOD score of 2.4 on chromosome 5 and 9 while two additional peaks at chromosome 6 and 19 were obtained with panel of $100 \mathrm{~K}$ markers (Fig. 2). Position of homozygous linkage region were as follows: \#5a, rs316574 (0-2304811 bp); \#5b, rs10062024-rs142796294 (3710645-4423695 bp); \#5c, rs379181-rs6873433 (5752026-6596404 bp); \#5d, rs2860357-rs13175954 (10950177-11723594 bp); \#6, rs2235233-rs3130814 (27279852-29196181); \#9, rs73654026-rs2487912 (94438287-100882881 bp):\#19a, rs35029254-rs17676218 (4874019-5288875 bp) and \#19b, rs67507307-rs 11671501 (13429114-14707030 bp) with cytoband 5p15.33 (2.3Mb), 5 p15.33 (0.71Mb), 5p15.32-p15.31 (0.84Mb), 5p15.2 $(0.77 \mathrm{Mb}), 6 \mathrm{p} 22.1(1.92 \mathrm{Mb}), 9 \mathrm{q} 22.31-\mathrm{q} 22.33(6.44 \mathrm{Mb})$, $19 \mathrm{p} 13.3 \quad(0.41 \mathrm{Mb})$ and $19 \mathrm{p} 13.2-\mathrm{p} 13.12 \quad(1.28 \mathrm{Mb})$. 
Exome sequencing data was filtered and scrutinized for causative variants by setting the minimal allele frequency to $<0.01$. This revealed a nonsense mutation in BICD2 (NM_001003800.1; c.204T $>$ G) which was located on chromosome 9 linkage region 9q22.31-q22.33 (6.44 $\mathrm{Mb})$. This variant in exon 1 changes a glutamic acid codon into a premature stop codon (p. Glu68*) (Fig.3A, 3B) and thus, was predicted to result in a severely truncated protein, if translated. Mutation taster (http://www.mutationtaster. org/) and CADD score (https://cadd.gs.washington. edu/score) predicted it to be pathogenic. This variant of $B I C D 2$ was not found in databases like dbSNP150, 1000 Genomes (build 20110521), in-house database at Cologne Center for Genomics with $>1,600$ exomes, ExAC (http:// exac.broadinstitute.org/), and gnomAD (https://gnomad. broadinstitute.org/).

A

B
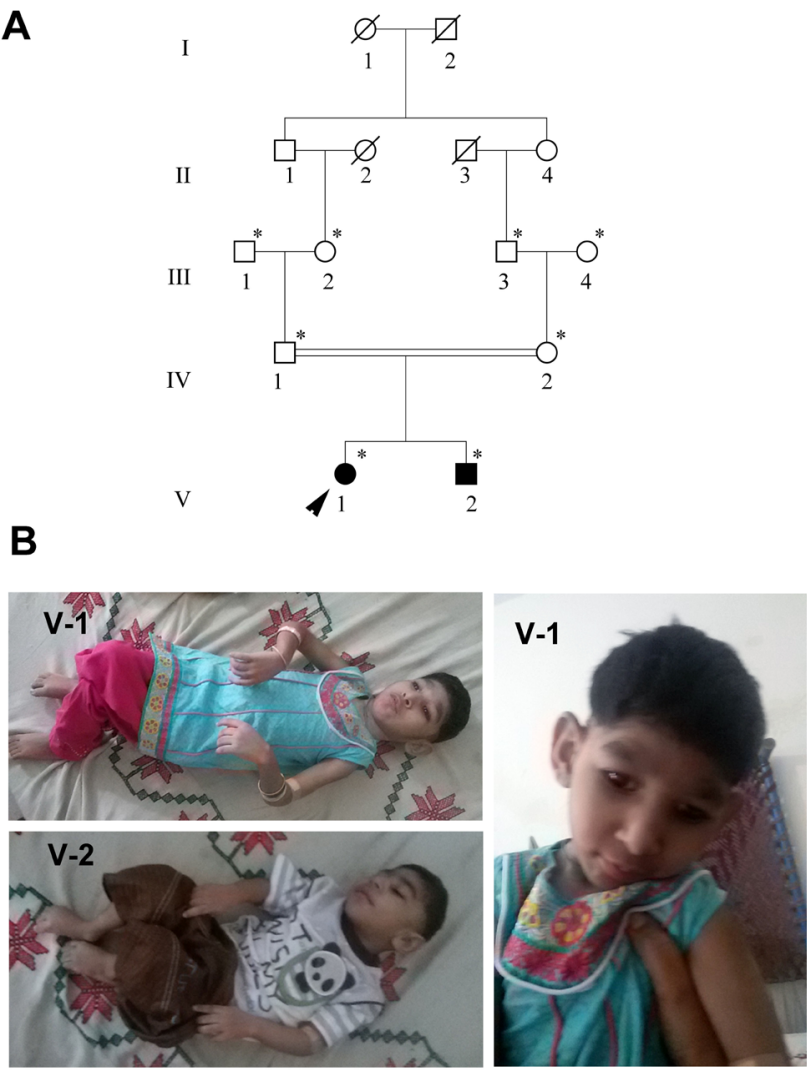

Fig. 1. Pedigree and clinical features. (A) Pedigree of family indicating second grade consanguinity and autosomal recessive mode of inheritance. Individuals with DNA samples available for genetic analyses are marked with asterisks. Arrowheads point to individual for whom whole exome sequencing was performed. (B) Clinical photographs of the affected members V-1 and V-2 showing stiffness and spasticity of upper as well as lower limbs and microcephalic feature.
Segregation analysis

Targeted Sanger sequencing results showed that this variant segregated with the phenotype in the expected autosomal recessive mode of inheritance, as all affected individuals were homozygous and carriers were heterozygous (Fig. 3C).

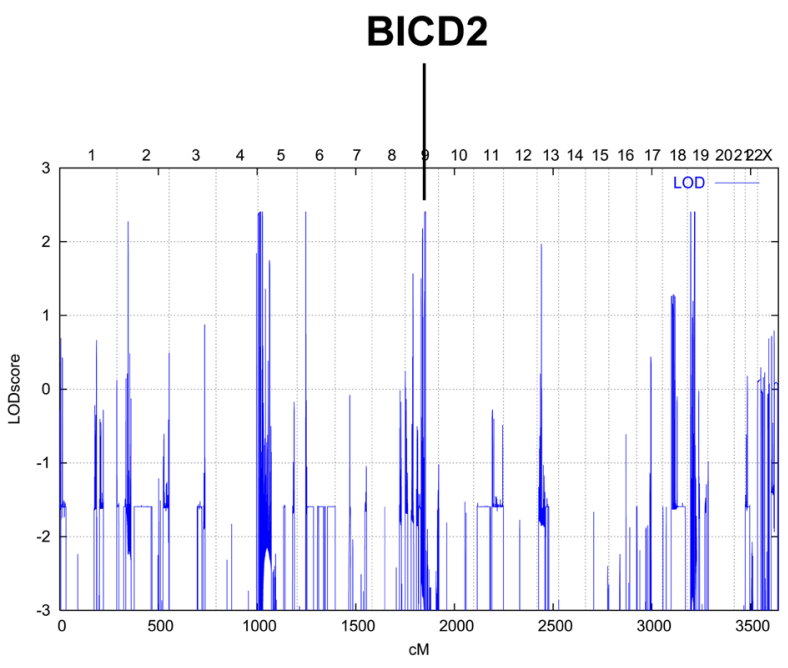

Fig. 2. Genome-wide scans for homozygous regions using $100 \mathrm{~K}$ marker panel and multipoint LOD score graphs generated with ALLEGRO. Linkage region on chromosome 9 harboring BICD2 is indicated.

\section{DISCUSSION}

We have identified a novel protein truncation mutation in BICD2. Human BICD2 (OMIM 609797) is a homologue of Drosophila bicaudal D (BicD). It encodes a ubiquitously expressed translational product of $96 \mathrm{kDa}$. BICD2 is a motor-adaptor protein that links the dynein motor complex to various cargoes. Five coiled coil regions constitute this highly conserved protein. The N-terminal $\mathrm{CC} 1$ domain interacts with dynein-dynactin complex, C-terminal CC3 domain links to RAB6A that localizes to Golgi apparatus and cytoplasmic vesicles while central CC2 domain binds to a kinesin KIF5A (Fig. 3B) (Matanis et al., 2002). It is proposed that when C-terminal region binds to the cargo, the $\mathrm{N}$-terminal part becomes available to bind to dynein and induces minus end directed transport (Hoongenraad et al., 2001). Intramolecular interactions between $\mathrm{N}$-termninal and $\mathrm{C}$-terminal domain were also demonstrated by Hoongenraad and colleagues suggesting that they have regulatory feature in accurate cellular trafficking by BICD2. So far, 25 mutations have been reported in $B I C D 2$ encompassing the entire coding sequence generating a spectrum of motor neuron ailments 
particularly autosomal dominant spinal muscular atrophy type 2 (SMALD2), amyotrophic lateral sclerosis and hereditary spastic paraplegia. So far only two families of HSP are reported inherited one by autosomal recessive and other by dominant mode. Three families of DCSMA+UMN (upper motor neuron) affected features inherited both by dominant and recessive mode were reported. Oates et al. 2013 identified missense mutation [c.1523A $>C$ ( $p$. Lys508Thr)] in one family of HSP penetrating dominantly with adult onset and three families of DCSMA+UMN affected features with onset by birth, all located in first two coiled coil regions. Transgenic investigations with two mutant BICD2 $2^{\text {p.Ser107Leu }}$ and BICD2 $2^{\text {p.Arg501Pro }}$ demonstrated increased binding affinity between BICD2 and dyneindynactin complex with perturbed cellular trafficking process leading to impaired motor neurons. A homozygous missense mutation [c.1823G $>$ A, (p.Ser608Leu)] was identified in family of pure HSP with autosomal recessive mode of inheritance and adult onset by implicating exome sequencing and HSPome network (Novarino et al., 2014).

The homozygous mutation in our family $(\mathrm{c} .204 \mathrm{~T}>\mathrm{G})$ causes the insertion of a premature stop codon in the open reading frame, predicted to results in a truncated protein of 68 amino acids (p.Glu68*). This nonsense mutation disrupts the first coiled coil domain depleting most of the protein. It is highly probable that the variant results in nonsense-mediated decay of mRNA and ultimately complete loss of protein. Phenotypic features presented by affected kindred of HSP family reported by Oats et al., 2013 were the lower limb spasticity and hyperreflexia. Contractures, weakness and wasting of muscles were progressive and less severe. Members of our family had upper and lower limbs spasticity, dysphagia, cognitive impairment and seizures episodes. Patients were bed ridden. They presented severe microcephaly with hearing and speech impairment. Bicd2 knockout mice developed disorganized cerebral cortex and the cerebellum due to impaired radial neuronal migration (Jaarsma et al., 2014). Thus, we can speculate that depletion of BICD2 in patients caused the deficit in neuronal cell migration to cortical plate, developing microcephaly phenotype. Golgi fragmentation, disturbed retrograde transport and axonal neurofilament swelling were observed in neurons of transgenic mice model expressing the $\mathrm{N}$-terminal region (Teuling et al., 2008). BICD2 depletion cause retarded dynein and kinesin dependent movement proving that both retrograde and anterograde trafficking along microtubules essentially requires BICD2 (Neveling et al., 2013; Oates et al., 2013; Peeters et al., 2013). BICD2 has crucial role in neuronal development. Outgrowth of neurite extensions during neuronal differentiation requires accurate anterograde secretory trafficking (Pfenninger, 2009).
However, recently it has been demonstrated that different heterozygous mutations in BICD2 irrespective of their position on gene, contribute to the stability of microtubules on motor neurons leading to abnormal axonal branching and reduced neuromuscular junctions (NMJ) justifying the neurological nature of disease (Martinez Carrera et al., 2018).

A

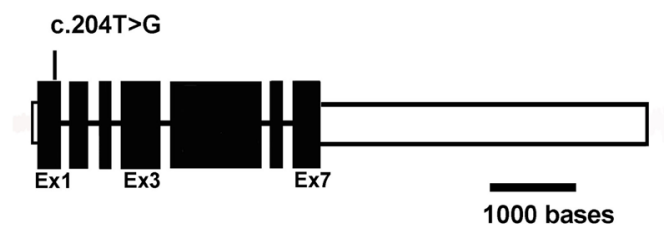

B

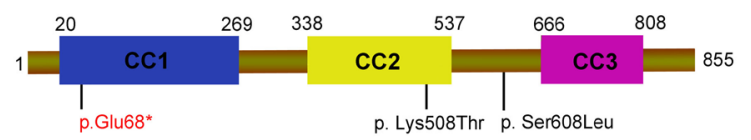

C
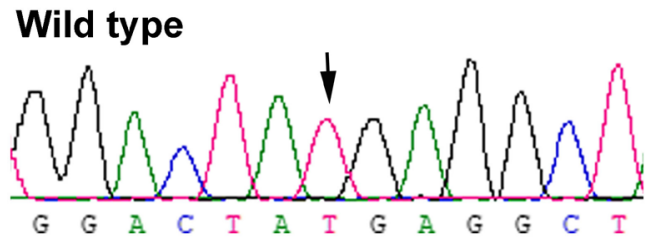

Mutant (c.204T>G: p.Glu68*)

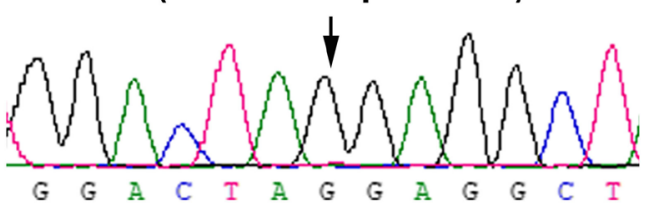

Hetrozygous Carrier

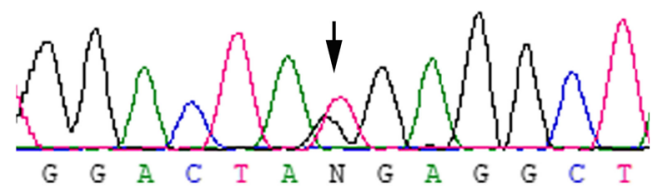

Fig. 3. Mutation identification (A) BICD2 gene structure showing 7 exons. The mutation identified is indicated above in exon1, unfilled boxes at the start and end of the gene represents UTR regions. (B). BICD2 protein structure ( 855 amino acids). Domains are indicated by the specified color. $\mathrm{N}$-terminal $\mathrm{CC} 1$ domain interacts with dynein-Dynactin complex, $\mathrm{CC} 2$ central domain interacts with kinesin protein and $\mathrm{CC} 3$ at $\mathrm{C}$-terminal interacts with RAB6A, RNBAP2. Variants identified in HSP families so far are shown below, mutation identified in this study is in red, while text in black indicates variants identified earlier. (C). Chromatograms showing mutation in patient, heterozygous carrier along with wild type control. 
So far only few missense mutations in BICD2 with relation to HSP with adult onset have been reported. We report the first nonsense mutation (c.204T>G; p. Glu68*) with severe phenotypic and clinical features and onset by birth. Further quantitative expression analysis and functional exploration of this variant can clearly explain the genotype-phenotype correlation.

\section{CONCLUSION}

Genetic exploration of HSP family with complex clinical features revealed a novel nonsense mutation in $B I C D 2$ gene. Mutational screening showed that insertion of premature stop codon after 68 amino acids yields extremely truncated protein product. We can speculate that the truncated protein product caused the deficit in normal neuronal development and function. Identification of this novel mutation has increased the mutational spectra of $B I C D 2$ gene but further functional exploration is required to develop a phenotype genotype correlation.

\section{ACKNOWLEDGEMENTS}

Funding was received from the Higher Education Commission of Pakistan (S.R). We thank the family who participated in this study; and Cologne Center for Genomics team for technical help. We thank Dr. Sadaf Naz for reviewing the manuscript before submission.

\section{Statement of conflict of interest}

The author declares that they have no conflict of interests .

\section{REFERENCES}

Ahmad, I., Baig, S.M., Abdulkareem, A.R., Hussain, M.S., Sur, I., Toliat, M.R., Nürnberg, G., Dalibor, N., Moawia, A., Waseem, S.S. and Asif, M., 2017. Genetic heterogeneity in Pakistani microcephaly families revisited. Clin. Genet., 92: 62-68. https:// doi.org/10.1111/cge.12955

Blackstone, C., O'kane, C.J. and Reid, E., 2011. Hereditary spastic paraplegias: membrane traffic and the motor pathway. Nature Rev. Neurosci., 12:3142. https://doi.org/10.1038/nrn2946

Finsterer, J., Löscher, W., Quasthoff, S., Wanschitz, J., Auer-Grumbach, M. and Stevanin, G., 2012. Hereditary spastic paraplegias with autosomal dominant, recessive, X-linked, or maternal trait of inheritance. J. Neurol. Sci., 318:.1-18. https://doi. org/10.1016/j.jns.2012.03.025

Giudice, T.L., Lombardi, F., Santorelli, F.M., Kawarai,
T. and Orlacchio, A., 2014. Hereditary spastic paraplegia: clinical-genetic characteristics and evolving molecular mechanisms. Exp. Neurol., 261:.518-539. https://doi.org/10.1016/j. expneurol.2014.06.011

Gudbjartsson, D.F., Jonasson, K., Frigge, M.L. and Kong, A., 2000. Allegro, a new computer program for multipoint linkage analysis. Nature Genet., 25:12. https://doi.org/10.1038/75514

Helms, C., 1990. Salting out Procedure for Human DNA extraction. The Donis-Keller Lab-Lab Manual Homepage. http://hdklab.wustl.edu/lab_manual/ dna/dna2.html.

Hoogenraad, C.C., Akhmanova, A., Howell, S.A., Dortland, B.R., De Zeeuw, C.I., Willemsen, R., Visser, P., Grosveld, F. and Galjart, N., 2001. Mammalian Golgi-associated Bicaudal-D2 functions in the dynein-dynactin pathway by interacting with these complexes. EMBO $J$., 20: 4041-4054. https://doi.org/10.1093/ emboj/20.15.4041

Jaarsma, D., Van Den Berg, R., Wulf, P.S., Van Erp, S., Keijzer, N., Schlager, M.A., De Graaff, E., De Zeeuw, C.I., Pasterkamp, R.J., Akhmanova, A. and Hoogenraad, C.C., 2014. A role for Bicaudal-D2 in radial cerebellar granule cell migration. Nature Commun., 5: 3411. https://doi.org/10.1038/ ncomms 5380

Klebe, S., Stevanin, G. and Depienne, C., 2015. Clinical and genetic heterogeneity in hereditary spastic paraplegias: from SPG1 to SPG72 and still counting. Rev. Neurol., 171: 505-530. https://doi. org/10.1016/j.neurol.2015.02.017

Martinez Carrera, L.A., Gabriel, E., Donohoe, C.D., Hölker, I., Mariappan, A., Storbeck, M., Uhlirova, M., Gopalakrishnan, J. and Wirth, B., 2018. Novel insights into SMALED2: BICD2 mutations increase microtubule stability and cause defects in axonal and NMJ development. Human mol. Genet., 27: 1772-1784. https://doi.org/10.1093/ hmg/ddy086

Matanis, T., Akhmanova, A., Wulf, P., Del Nery, E., Weide, T., Stepanova, T., Galjart, N., Grosveld, F., Goud, B., De Zeeuw, C.I. and Barnekow, A., 2002. Bicaudal-D regulates COPI-independent Golgi-ER transport by recruiting the dynein-dynactin motor complex. Nature Cell Biol., 4: 986-992. https://doi. org/10.1038/ncb891

Nagle, D.L., Karim, M.A., Woolf, E.A., Holmgren, L., Bork, P., Misumi, D.J., McGrail, S.H., Dussault Jr, B.J., Perou, C.M., Boissy, R.E. and Duyk, G.M., 1996. Identification and mutation analysis 
of the complete gene for Chediak-Higashi syndrome. Nature Genet., 14:307-311. https://doi. org/10.1038/ng1196-307

Neveling, K., Martinez-Carrera, L.A., Hölker, I., Heister, A., Verrips, A., Hosseini-Barkooie, S.M., Gilissen, C., Vermeer, S., Pennings, M., Meijer, R. and te Riele, M., 2013. Mutations in BICD2, which encodes a golgin and important motor adaptor, cause congenital autosomal-dominant spinal muscular atrophy. Am. J. Human Genet., 92: 946954. https://doi.org/10.1016/j.ajhg.2013.04.011

Novarino, G., Fenstermaker, A.G., Zaki, M.S., Hofree, M., Silhavy, J.L., Heiberg, A.D., Abdellateef, M., Rosti, B., Scott, E., Mansour, L. and Masri, A., 2014. Exome sequencing links corticospinal motor neuron disease to common neurodegenerative disorders. Science, 343: 506-511. https://doi. org/10.1126/science. 1247363

Oates, E.C., Rossor, A.M., Hafezparast, M., Gonzalez, M., Speziani, F., MacArthur, D.G., Lek, M., Cottenie, E., Scoto, M., Foley, A.R. and Hurles, M., 2013. Mutations in BICD2 cause dominant congenital spinal muscular atrophy and hereditary spastic paraplegia. Am. J. Human Genet., 92: 965973. https://doi.org/10.1016/j.ajhg.2013.04.018

O'Connell, J.R. and Weeks, D.E., 1998. PedCheck: a program for identification of genotype incompatibilities in linkage analysis. $\mathrm{Am}$. J. Human Genet., 63: 259-266. https://doi. org/10.1086/301904

Parodi, L., Fenu, S., Stevanin, G. and Durr, A., 2017. Hereditary spastic paraplegia: More than an upper motor neuron disease. Rev. Neurol., 173: 352-360. https://doi.org/10.1016/j.neurol.2017.03.034

Peeters, K., Litvinenko, I., Asselbergh, B., AlmeidaSouza, L., Chamova, T., Geuens, T., Ydens, E., Zimoń, M., Irobi, J., De Vriendt, E. and De Winter, V., 2013. Molecular defects in the motor adaptor
BICD2 cause proximal spinal muscular atrophy with autosomal-dominant inheritance. Am. J. Human Genet., 92: 955-964. https://doi.org/10.1016/j. ajhg.2013.04.013

Pfenninger, K.H., 2009. Plasma membrane expansion: a neuron's Herculean task. Nature Rev. Neurosci., 10: 251-261. https://doi.org/10.1038/nrn2593

Rossor, A.M., Oates, E.C., Salter, H.K., Liu, Y., Murphy, S.M., Schule, R., Gonzalez, M.A., Scoto, M., Phadke, R., Sewry, C.A. and Houlden, H., 2014. Phenotypic and molecular insights into spinal muscular atrophy due to mutations in BICD2. Brain, 138: 293-310. https://doi.org/10.1093/brain/awu356

Salinas, S., Proukakis, C., Crosby, A. and Warner, T.T., 2008. Hereditary spastic paraplegia: clinical features and pathogenetic mechanisms. Lancet Neurol., 7: 1127-1138. https://doi.org/10.1016/ S1474-4422(08)70258-8

Synofzik, M., Martinez-Carrera, L.A., Lindig, T., Schöls, L. and Wirth, B., 2014. Dominant spinal muscular atrophy due to BICD2: a novel mutation refines the phenotype. J. Neurol. Neurosurg. Psychiat., 85: 590-592. https://doi.org/10.1136/ jnnp-2013-306777

Szczepanski, S., Hussain, M.S., Sur, I., Altmüller, J., Thiele, H., Abdullah, U., Waseem, S.S., Moawia, A., Nürnberg, G., Noegel, A.A. and Baig, S.M., 2016. A novel homozygous splicing mutation of CASC5 causes primary microcephaly in a large Pakistani family. Human Gene., 135: 157-170.

Teuling, E., van Dis, V., Wulf, P.S., Haasdijk, E.D., Akhmanova, A., Hoogenraad, C.C. and Jaarsma, D., 2008. A novel mouse model with impaired dynein/dynactin function develops amyotrophic lateral sclerosis (ALS)-like features in motor neurons and improves lifespan in SOD1-ALS mice. Human mol. Genet., 17: 2849-2862. https:// doi.org/10.1093/hmg/ddn182 